\title{
Proximate mechanisms of the differences in reproductive success of males bearing different alleles of Pgdh - a gene involved in a sexual conflict in bulb mite
}

\author{
ANNA M. SKWIERZYŃSKA \& AGATA PLESNAR-BIELAK \\ Institute of Environmental Sciences, Jagiellonian University, Kraków, Poland
}

Keywords:

interlocus sexual conflict;

male mating behavior;

male reproductive success;

Pgdh;

phosphogluconate dehydrogenase;

sperm competitiveness.

\begin{abstract}
Enzyme polymorphism in phosphogluconate dehydrogenase (Pgdh) is a striking example of single gene polymorphism involved in sexual conflict in bulb mite Rhizoglyphus robini. Males homozygous for the S Pgdh allele were shown to achieve higher reproductive success than FF homozygous males, while negatively influencing fecundity of their female partners. Here, we investigate proximate mechanisms responsible for the increased reproductive success of SS males and find that the S allele is associated with shorter time until copulation, higher copulation frequency and increased sperm production. We also show that Pgdh alleles are probably codominant, with SS males gaining the highest reproductive success, FF males - the lowest - and FS-heterozygous males taking an intermediate position in all fitness parameters differentiating males of different genotypes. Additionally, we confirm the negative effect that $S$-bearing males impose on the fecundity of females they mate with, showing a clear pattern of interlocus sexual conflict. We discuss that this effect is probably associated with increased copulation frequency. Whereas, contrary to what we have predicted, the $\mathrm{S}$ allele does not cause increased general male mobility, we speculate that the $S$ allele-bearing males are more efficient in forcing copulation and/or detecting females.
\end{abstract}

\section{Introduction}

Evolutionary interests of males and females are usually not the same, leading to different reproductive roles of the sexes. This results in sexual conflict, where a trait that is beneficial for one sex is detrimental to the other (Parker, 1979). Sexual conflict may take one of two forms. Intralocus sexual conflict is expressed when the direction of selection on a shared trait changes depending on the sex (Chippindale et al., 2001; Bonduriansky \& Chenoweth, 2009). Interlocus sexual conflict, on the other hand, emerges when a trait that increases male reproductive success simultaneously reduces the fitness of their female partners (Parker, 1979; Chapman et al., 2003; Arnqvist \& Rowe, 2005). This is because selection

Correspondence: Anna Maria Skwierzyńska, Institute of Environmental Sciences, Jagiellonian University, Gronostajowa 7, 30-387 Kraków, Poland.

Tel.: + 4812664 5154; fax. + 48126646912 ;

e-mail: anna.skrzynecka@doctoral.uj.edu.pl on males often favours traits that increase male sperm competitiveness (Chapman et al., 1995), facilitate coercing females into mating (Arnqvist \& Rowe, 2005) or reduce the re-mating rate of the male's partners (Rice, 1996) at the expense of female lifetime reproductive success (Rice, 1996; Chapman et al., 1998, 2003; Arnqvist \& Rowe, 2005; Edward et al., 2011; Brommer et al., 2012).

Perhaps the best-described example of interlocus sexual conflict is the existence of over 80 (Swanson et al., 2001) seminal fluid proteins transferred to females while mating in Drosophila melanogaster (Chapman et al., 1995), which influence female behaviour and physiology. These proteins allow the males to up-regulate the females' egg-laying rate (Chapman et al., 2003; Liu \& Kubli, 2003); reduce their re-mating rate (Chapman et al., 2003; Liu \& Kubli, 2003); and manipulate their sperm retention and storage (Avila et al., 2010), feeding rate (Carvalho et al., 2006), food preference (Ribeiro \& Dickson, 2010) and water balance (Cognigni et al., 
2011), eventually shortening the females' lifespan and lowering their fitness (Chapman et al., 1995, 2000; Neubaum \& Wolfner, 1999; Heifetz et al., 2000; Chapman, 2001; Lung et al., 2002).

Because sexual conflict originates from anisogamy, it is commonly observed in a wide range of sexually reproducing species (reviewed in Chapman et al., 2003; Arnqvist \& Rowe, 2005). Both intra- and interlocus sexual conflict are also believed to play a substantial role in a number of evolutionary processes, with the latter being potentially important in the evolution of reproductive isolation and speciation (Rice $\delta$ Holland, 1997; Parker \& Partridge, 1998; Rice, 1998; reviewed in Gavrilets \& Hayashi, 2005; Gavrilets, 2014), driving patterns of genetic polymorphism in females (Franke et al., 2002; Gavrilets \& Waxman, 2002; Gavrilets \& Hayashi, 2005; Härdling \& Bergsten, 2006; Hayashi et al., 2007) and influencing the evolution of ageing and senescence (Promislow, 2003; Bonduriansky et al., 2008; Fricke et al., 2013; see also Priest et al., 2008). In extreme cases, sexual conflict can even lead to the extinction of a population (Kokko \& Brooks, 2005).

Given its ubiquity and importance in evolution, it is extremely surprising that there are almost no examples where variation in male reproductive success and in male effects on female fitness can be linked with the exact mechanisms of sexual conflict and genetic polymorphism associated with it (but see Herndon $\&$ Wolfner, 1995; Filice \& Long, 2016). Here, we investigate proximate mechanisms of sexual conflict that are associated with phosphogluconate dehydrogenase (Pgdh) polymorphism in the bulb mite Rhizoglyphus robini (Acari, Acaride), where the alternative alleles cause large differences in male reproductive success, affecting male sperm competitiveness. Furthermore, males bearing the 'winning' form of Pgdh have detrimental effects on their female partners' fitness, providing a spectacular example of a gene involved in the sexual conflict (Konior et al., 2006; Łukasik et al., 2010).

Phosphogluconate dehydrogenase (Pgdh) is an enzyme in the pentose phosphate pathway, which is an alternative to the glycolysis pathway for converting glucose. Unlike in glycolysis, no ATP is generated in this pathway. Instead, the pentose phosphate pathway produces NADPH which is necessary for reduction transformations such as fatty acids synthesis (Murray et al., 2003). Furthermore, the pentose phosphate pathway is a source of ribulose-5-phosphate used for nucleotide biosynthesis (Murray et al., 2003). Thus, by affecting the rate of nucleic acid synthesis, different Pgdh forms may differentially affect cell division and spermatogenesis rates. Polymorphisms between Pgdh variants have been reported to influence the phenotype in plants and animals by affecting such components of fitness as the probability of survival and salinity resistance in the wild (Stockwell \& Mulvey, 1998; Conte et al., 2003).
Konior et al. (2006) have demonstrated that males homozygous for the Pgdh form which moves slower during electrophoresis (SS) have ca. 50\% advantage in sperm competition with males homozygous for the Pgdh form which moves faster during electrophoresis (FF). Furthermore, they have shown that females mated with FF males have ca. 30\% higher fecundity rate than females mated with SS males. These observations are consistent with the predictions of antagonistic evolution models, according to which males achieving the highest reproductive success (owing to their ability to manipulate female preferences, behaviour or physiology) should impose the most detrimental effect on females (Holland \& Rice, 1998).

Neither the mechanism of winning sperm competition nor of males' detrimental effect on the fitness of their female partners is yet known. The main aim of this study was to attempt to reveal these mechanisms. We predict that males with SS genotype in Pgdh genes might produce a higher number of sperm cells, which could be the explanation for their higher success rate in sperm competition. Previous studies also indicate that frequent copulations decrease female fitness (Kołodziejczyk \& Radwan, 2003), which leads us to a prediction that SS males are more mobile and, hence, copulate more frequently, than FF males. This could be a possible mechanism of the detrimental effect on females imposed by SS males.

\section{Materials and methods}

\section{Pgdh genotyping}

Using transcriptomic data generated in another project (Stuglik et al., 2014), we obtained a sequence of the Pgdh region for $R$. robini. Based on this 527-nucleotidelong sequence, we found four SNPs which were different between individuals with alternative enzyme forms. Using these sites, we designed PCR primers allowing to differentiate between Pgdh genotypes. However, none of the primer pairs allowed the differentiation of all possible Pgdh genotypes (SS, FS and FF). Thus, we decided to use the three primers enabling us to differentiate individuals possessing $\mathrm{F}$ allele ( $\mathrm{SF}$ and $\mathrm{FF}$ ) from those lacking it (SS). The following sequences were used: 5'-GCCATTTCAGCATTGGACTT-3', 5'-AATAGTTT TGTTCACACGTACCC-3' and 5'-AATAGTTTTGTTCACA CGTACCA-3'. To discriminate FF from SF genotypes, every individual whose genotype we were going to assess was mated with an individual from the base population in which the $S$ allele is fixed. Based on the presence of SS homozygotes in offspring, we were able to assess the genotype of the individual (for SF heterozygote, about half of the offspring were SS homozygotes; for FF homozygote, there were no SS homozygotes among offspring). 


\section{Establishment of experimental population with increased frequency of $F$ allele}

Ten natural bulb mite populations from different locations were tested for Pgdh polymorphism. Twenty individuals from each of these populations were genotyped, and both alleles were detected in one of them-a population from Mielec, Poland-which was used to establish an experimental population for further experiments. In this population, six individuals (of 20 that were genotyped) were shown to possess at least one F allele. We collected ca. 100 individuals from the Mielec population and allowed them to propagate in standard laboratory conditions $\left(24^{\circ} \mathrm{C},>90 \%\right.$ humidity, constant darkness, powdered yeast provided ad libitum as a food source) for one month, which is enough for mites to complete two generations. After this time, we assessed the Pgdh genotype of 40 females (females are larger and, therefore, it is easier to extract DNA in quantities sufficient for PCR). We found that the proportion of $\mathrm{F}$ allele was $26 \%$ (23 SS individuals, $13 \mathrm{SF}$ individuals and four FF individuals).

Previous research (Konior et al., 2006) showed that the $\mathrm{F}$ allele is removed by selection from laboratory populations very quickly because of the higher reproductive success of males with the $\mathrm{S}$ allele. To obtain a population with a high frequency of the $\mathrm{F}$ allele, we selected 100 pairs of individuals from our experimental population and allowed them to reproduce in individual vials, thereby eliminating reproductive competition between males. After 7 days, we assayed all parents for the presence of the $\mathrm{F}$ allele. In 36 pairs, at least one parent was the bearer of the $\mathrm{F}$ allele. To obtain an experimental population with an elevated proportion of the $\mathrm{F}$ allele, we used the offspring of these 36 pairs. We allowed such a population to expand freely for an additional month to obtain a sufficient number of individuals for the main experiments.

\section{Male mating behaviour and general mobility}

In the experiments, we used naïve males-males which were isolated and housed in individual vials before reaching adulthood and hence virgin. To obtain such males, we randomly selected 50 previously mated females from the experimental population and allowed them to lay eggs for 2 days in a common container. On the third day, the females were removed and the eggs were allowed to develop in the absence of adult individuals. After the emergence of tritonymphs (the last juvenile stage of the bulb mite), they were isolated to individual vials and allowed to reach the adult stage. Experimental observations were conducted 3-5 days after the adults emerged.

Each naïve male from the experimental population (117 males in total) was mated with a virgin female from the stock population (homozygous for the $\mathrm{S}$ allele) in a fresh vial. Such a pair was observed for $3 \mathrm{~h}$ under a stereomicroscope. To determine differences between males in mating behaviour, we noted latency to the first copulation and the number of copulations. Latency to the first copulation was treated as a proxy of male effectiveness in finding female partner. Number of copulations was predicted to vary between males differing in Pgdh genotype due to possible changes in metabolism. After observations, the pairs were left for additional 7 days for further observations.

To assess copulation frequency in longer periods of time, we checked all vials four times a day on days 2, 4 and 6 (checked every 2 h, in total, 12 checks per vial) and noted whether pairs were in copula or not. On those days, after the fourth observation, the pairs were moved to fresh vials to prevent high egg densities. All the vials were kept for further egg number assessment (see the next subsection).

To assess the general mobility of males, we observed each male for $5 \mathrm{~min}$ on days 3,5 and 7 and noted the amount of time for which the male was actively moving. If the male was in copula during our checks, we skipped such a vial and came back to it later, as we were interested in the amount of time that males spent voluntarily on seeking females and food. In further analysis, we used the mean male mobility during those three checks as a proxy of general male mobility which was predicted to be affected by male genotype.

After 7 days of interaction, the adults were removed. All the males were genotyped, whereas females were discarded. As we were interested in male genotypes and therefore needed the males' offspring, we transferred 14 eggs from each pair to a new container and allowed them to develop before genotyping. If there were less than 14 eggs, all eggs were left for further development. In nine cases of 117, females did not lay any eggs, so we excluded those observations from further analysis as we could not assess the male Pgdh genotype.

\section{Confirmation of negative influence of males with the $S$ allele on fecundity of females}

Previous research showed that males bearing the $\mathrm{S}$ form of Pgdh are harmful to females and reduce their fecundity in monogamous matings (Konior et al., 2006). We wanted to confirm these findings by assessing the female fecundity after 7 days of constant interaction with males differing in their Pgdh genotypes.

To assess female fecundity, we counted eggs laid by females used in the previous experiment after 7 days of interaction with a single male. Seven-day oviposition period is representative of a female's lifetime egg production, as oviposition rate is constant during the first 3 weeks when the females manage to lay the vast majority of their eggs (Konior et al., 2001; Tilszer et al., 2006). The cases in which female did not lay eggs (nine 
females of 117) were excluded from further analysis, as we could not differentiate male and female infertility in our design and were unable to assess the male genotype.

\section{Sperm production}

To assess the number of sperm produced by males differing in the Pgdh form, we mated males from the experimental population with five females each, to avoid male fertility limitation by female fecundity. Females of $R$. robini do not lay unfertilized eggs, so the number of laid eggs is a good estimation of the sperm amount transferred by a male (Radwan \& Siva-Jothy, 1996). To obtain same-age males, we selected 100 previously mated females from the experimental population and allowed them to lay eggs for $24 \mathrm{~h}$. After this period, females were removed and eggs were allowed to develop. After 14 days, when all individuals were mature and had time to interact, we isolated 63 males to individual vials to allow them to restore their sperm supplies. After 3 days, we mated each male with five virgin females from the stock population obtained by isolating tritonymphs and keeping them separately prior to the experiment. Such groups stayed together for 5 days, after which males were removed from the vials and females were left for another 2 days of oviposition. After 7 days, females were also removed and the number of eggs laid by the females was scored. Males were then genotyped for Pgdh.

\section{Statistics}

To test the influence of genotype on latency to first copulation, we used Kruskal-Wallis test owing to the abnormal distribution of the data. Analysis was carried out using observations for 108 males.

The influence of male genotype on the number of copulations during the continuous observation was assessed using Generalized Linear Model with the number of copulations as a dependent variable and male genotype as a fixed factor with a quasi-Poisson distribution of errors. Analysis was carried out using observations for 108 males.

Male genotype effects on mating frequency in a longer period of time were analysed using Ancova, with mating frequency (proportion of matings to all controls) as a dependent variable, male genotype as a fixed factor and male mean mobility as a covariate. Analysis was carried out using observations for 108 males.

Male genotype influence on female fitness was checked using anova with female fecundity as a dependent variable and male genotype as a fixed factor. Analysis was carried out using data for 108 pairs.

To test the influence of male genotype on the number of sperm produced, we used ANova with the number of eggs laid by females as a dependent variable and male genotype as a fixed factor. Analysis was carried out using data for 63 males.

To test for the differences between particular genotypes, we applied post hoc tests. The Nemenyi post hoc test was used for the analysis of latency to copulation. In all other cases, Tukey's HSD test was used.

All statistical analyses were performed in $\mathrm{R}$ version 3.4.1 (R Development Core Team, 2017). Normality of model residuals was inspected visually using Q-Q plots, and homoscedasticity of variance was ensured by inspecting plots of residuals vs. fitted values (Crawley, 2012).

\section{Results}

\section{Male mating behaviour and general mobility}

Latency to first copulation was significantly influenced by male genotype $\left(\chi^{2}=61.673\right.$, d.f. $=2, \quad \mathrm{~N}=108$, $P<0.001$, Fig. la). Post hoc analysis (Nemenyi test) revealed that all genotypes differed significantly from each other (FF-FS: $P=0.0007$, SS-FS: $P=0.0005$, FFSS: $P<0.001)$. As expected, SS males started copulations in the shortest time (mean $\pm \mathrm{SE}=1606.76 \pm$ $34.41 \mathrm{~s})$, FF males had the longest latency to first copulation $(2302.36 \pm 37.91 \mathrm{~s})$, and an intermediate result was observed with heterozygous males (1928.69 \pm $37.26 \mathrm{~s})$.

Furthermore, the number of copulations during constant observation was significantly influenced by male genotype $\left(F_{2,105}=5.454, P=0.005\right.$, Fig. lb $)$. Tukey's HSD test revealed that $S$-homozygous males copulated significantly more frequently than F-homozygous males $(P=0.003)$ with heterozygous males showing an intermediate frequency; however, difference between heterozygous males and both types of homozygotes was not significant (SS-FS: $P=0.256$, FF-FS: $P=0.253$ ).

Male genotype significantly influenced the frequency of copulations measured in longer periods of time $\left(F_{2,104}=6.169, \quad P=0.002\right.$, Fig. 2a). However, mean male mobility did not affect copulation frequency $\left(F_{1,104}=2.314, \quad P=0.131\right.$, Fig. $\left.2 b\right)$. Again, SS males had the highest frequency of copulations, FF males had the lowest, and FS males showed intermediate results. The difference between homozygotes was statistically significant $(P=0.002)$, whereas the differences between both types of homozygotes and heterozygotes were not (SS-FS: $P=0.069$, FF-FS: $P=0.489$ ).

\section{The influence of male genotype on fecundity of females}

Female fecundity was significantly altered by male Pgdh genotype $\left(F_{2,105}=9.296, P<0.001\right)$. Females mated to F-homozygous males laid the highest number of eggs and those mated with S-homozygotes laid the lowest number of eggs. Females mated with heterozygotes 

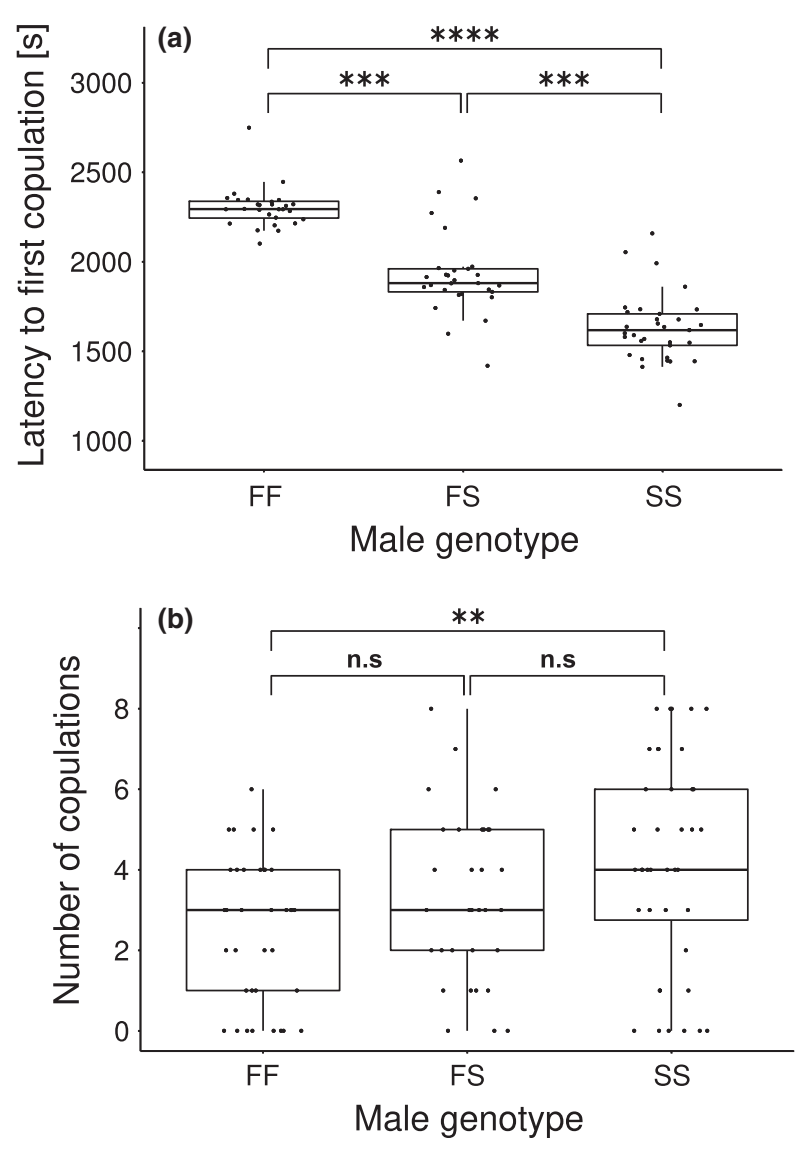

Fig. 1 Latency to copulation (a) and number of copulations during constant observation period (b) for males differing in Pgdh form. Raw data and boxplots are presented. Asterisks represent differences between genotypes revealed in post hoc tests [Nemenyi test (a) and Tukey's range test (b)]. Model details are presented in Materials and Methods section. Significance levels are $<0.01(* *)$, $<0.001(* * *),<0.0001(* * * *)$. Abbreviation n.s stands for nonsignificant difference.

showed intermediate fecundity (Fig. 3). The differences in fecundity between females mated to FF males and FS males and between females mated to FF males and SS males were statistically significant ( $P=0.009$ and $P<0.001$, respectively), whereas the difference between females mated to SS males and FS males was not significant $(P=0.614)$.

\section{Sperm production}

Sperm production was significantly influenced by male genotype $\left(F_{2,60}=5.624, P=0.006\right)$ with SS males producing the highest number of sperm and FF males the lowest number. FS males again showed intermediate values (Fig. 4). However, only the difference between two types of homozygotes was statistically significant $(P=0.004)$, whereas the others were not $(\mathrm{FF}-\mathrm{FS}$ : $P=0.159$, SS-FS: $P=0.331$, Fig. 4).
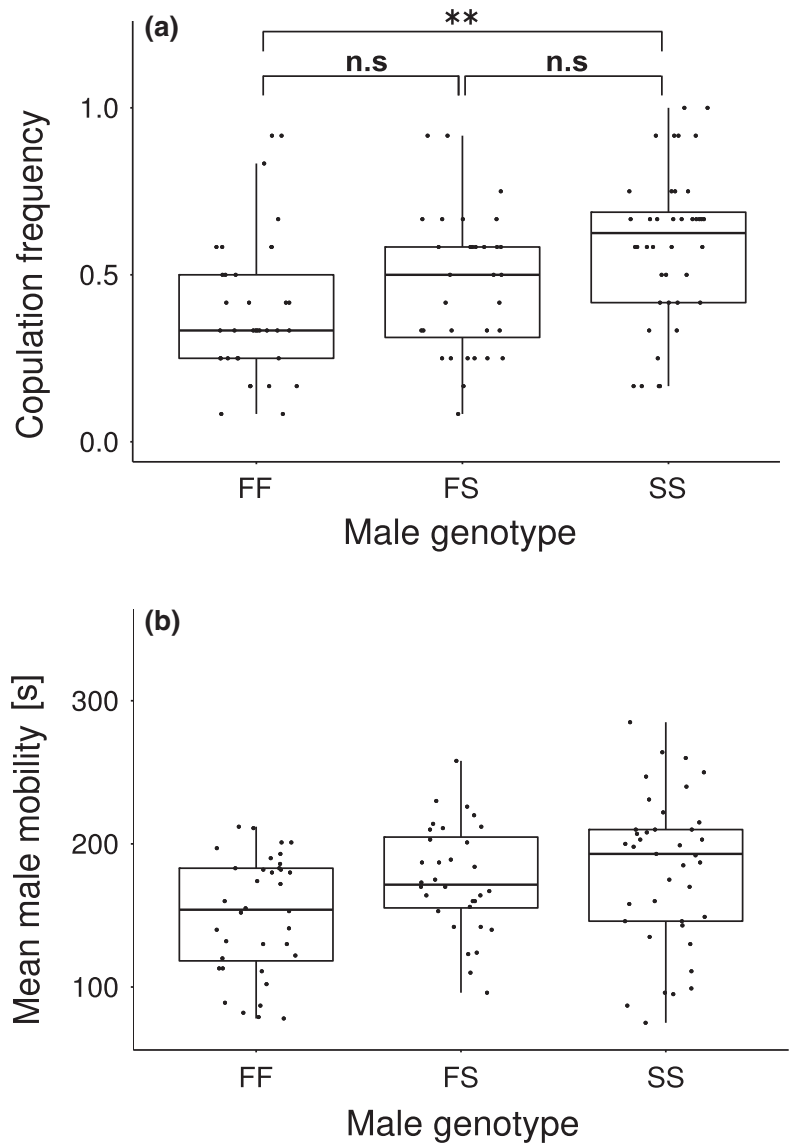

Fig. 2 Copulation frequency (a) and mean male mobility (b) for males differing in the Pgdh form during the 7-day trial. Raw data and boxplots are presented. Asterisks on panel A represent statistically significant differences between genotypes. Model details are presented in Materials and Methods section. Significance level is $<0.01\left(^{* *}\right)$. Abbreviation n.s stands for nonsignificant difference.

\section{Discussion}

Even though interlocus sexual conflict is predominant in nature, surprisingly few studies have identified its genetic background. Thus, enzyme polymorphism in phosphogluconate dehydrogenase (Pgdh) is a striking example of single gene polymorphism involved in a sexual conflict. Nevertheless, proximate mechanisms underlying its effect on male and female fitness have not been identified so far. In the present study, we investigate mechanisms explaining the increased reproductive success of males bearing the S allele of Pgdh and their detrimental effects on female fitness. We demonstrate that Pgdh genotype affects several male traits, including the production of sperm and behavioural traits.

We show that the $S$ allele of Pgdh is responsible for increased production of sperm. This confirms earlier 


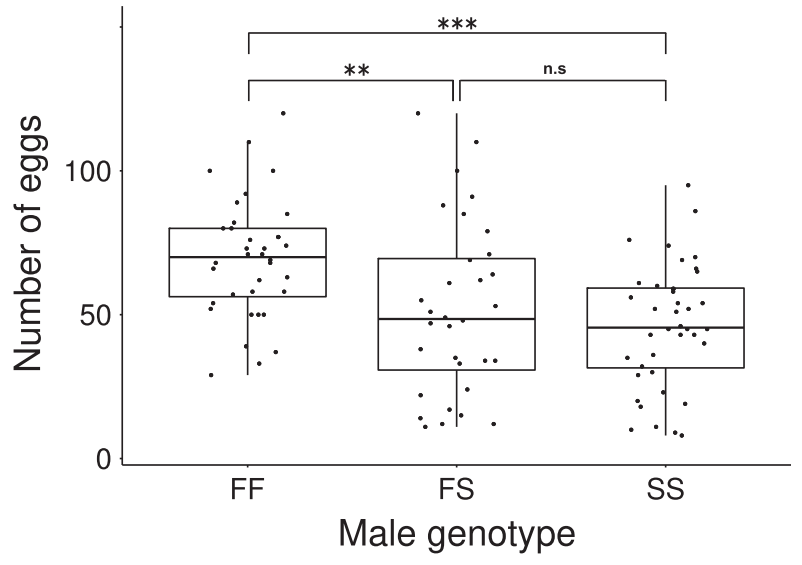

Fig. 3 Fecundity of females mated to males differing in the Pgdh form during the 7-day trial. Raw data and boxplots are presented. Asterisks represent statistically significant differences between genotypes. Model details are presented in Materials and Methods section. Significance levels are $<0.01\left(^{* *}\right)$ and $<0.001\left(^{(* *}\right)$. Abbreviation n.s stands for nonsignificant difference.

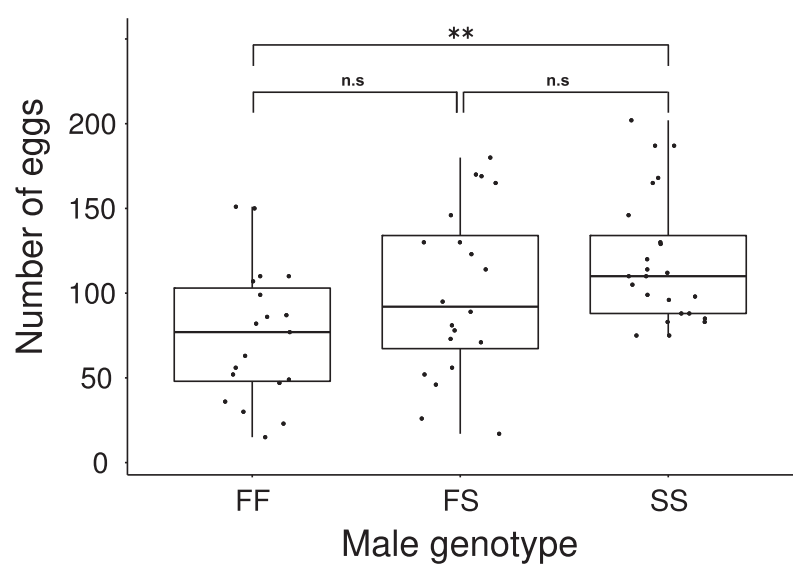

Fig. 4 Number of eggs fertilized by males differing in the Pgdh form (Rhizoglyphus robini females do not lay unfertilized eggs; hence, number of laid eggs is a good proxy for number of produced sperm). Raw data and boxplots are presented. Asterisks represent statistically significant differences between genotypes. Model details are presented in Materials and Methods section. Significance levels is $<0.01(* *)$. Abbreviation n.s stands for nonsignificant difference.

suggestions that higher sperm production is a possible mechanism of elevated sperm competitiveness of SS males in comparison with FF males reported in previous studies (Konior et al., 2006; Łukasik et al., 2010). However, it is uncertain whether this is the only factor that differentiates sperm produced by different Pgdh genotype males. For example, our design does not allow to rule out the possibility that increased sperm production by SS males is accompanied by increased sperm quality (e.g. large size (Radwan, 1996) or higher viability of sperm). Further investigation of this problem could possibly involve measurements of various sperm parameters.

We also demonstrate that Pgdh genotype influences male behaviour, being another cause of the different reproductive success of males with different Pgdh variants. Males bearing the $S$ allele are more efficient in initiating copulation and copulate more frequently than those lacking it. Frequent copulations may be associated with deleterious effect imposed by SS males on their female partners (Konior et al., 2006; this study) as multiple matings have been shown to be deleterious to females in $R$. robini (Kołodziejczyk \& Radwan, 2003) and some other species. For example, in Drosophila grimshawi, males exhibiting higher courtship vigour have a higher probability of mating, but they also negatively affect female fecundity (Droney, 2003). Similarly, in $D$. melanogaster, repeated mating is costly to females (Chapman et al., 1995). Larger males, which mate more often, cause the highest reduction in female fitness (Friberg \& Arnqvist, 2003). Interestingly, in our study, increased frequency of copulations of SS males was not associated with elevated general mobility of these males, ruling out the possibility that SS males mate more frequently just because they move more and simply encounter females more often. It may suggest that males with an advantageous form of Pgdh are more effective in detecting females and/or in forcing females to copulate. However, verifying these hypotheses needs further experimental effort.

Our results suggest codominance of Pgdh alleles. Shomozygous males start copulation sooner, copulate more frequently and produce more sperm that F-homozygous males, whereas SF heterozygotes show an intermediate value in all of these measurements. Although differences between all three genotypes were statistically significant only in the case of latency to first copulation, our results show a clear and repeatable pattern. Similarly, in an earlier study, Eukasik et al. (2010) found that sperm competitiveness of FS males was lower than that of FF males and higher than that of SS males. However, the difference between FS and both homozygotes was also not statistically supported.

The fact that the effects of both alleles are present in heterozygotes (codominance) clearly affects the dynamics of the $\mathrm{F}$ and the $\mathrm{S}$ allele frequencies. As the $\mathrm{F}$ variant quickly declines and becomes lost from populations under laboratory conditions (Konior et al., 2006; Łukasik et al., 2010), the S allele advantage in male competitiveness has to outweigh the costs associated with fecundity decline of their partners. This is often the case in nonmonogamous populations in which males mate with multiple females. As mites are highly promiscuous, copulations are frequent in large, dense laboratory conditions and males bearing the $S$ allele are indeed 
likely to rapidly outcompete rivals. Interestingly, in natural populations of $R$. robini, both alleles might be maintained. Konior et al. (2006) reported two populations in which $\mathrm{F}$ allele proportion was 0.22 and 0.34 , respectively. It was also shown that alleles frequencies vary substantially among populations and years (Eukasik et al., 2010) although exact mechanism driving those differences was not shown. It would be interesting to see whether females are able to evolve counter-adaptations reducing harm induced by $\mathrm{S}$ males on their fecundity (as predicted by the theory of sexual conflict; Rice, 1998; Arnqvist \& Rowe, 2002; Wigby \& Chapman, 2004) or whether they could evolve a preference for FF males in Pgdh polymorphic populations.

The mechanisms maintaining Pgdh polymorphism in nature are still a puzzle and need investigation. Further studies of this problem should shed more light on the conditions under which polymorphism of genes under sexual conflict (and thus sexual conflict itself) is maintained and on the forces sustaining genetic variation in general.

\section{Acknowledgments}

We thank two anonymous referees for their comments on the previous version and constructive criticism. We thank Prof Jacek Radwan for his ideas and invaluable help in planning this research, Adam Stefaniak and Richa Joag for their comments on this manuscript. This work was supported by the Polish National Science Centre (NCN) UMO-2011/03/N/NZ8/00016.

\section{References}

Arnqvist, G. \& Rowe, L. 2002. Antagonistic coevolution between the sexes in a group of insects. Nature 415: 787789.

Arnqvist, G. \& Rowe, L. 2005. Sexual Conflict. Princeton University Press, Princeton, NJ.

Avila, F.W., Ravi Ram, K., Bloch Qazi, M.C. \& Wolfner, M.F. 2010. Sex peptide is required for the efficient release of stored sperm in mated Drosophila females. Genetics 186: 595600 .

Bonduriansky, R. \& Chenoweth, S.F. 2009. Intralocus sexual conflict. TREE 24: 280-288.

Bonduriansky, R., Maklakov, A.A., Zajitschek, F. \& Brooks, R. 2008. Sexual selection, sexual conflict and the evolution of ageing and life span. Funct. Ecol. 22: 443-453.

Brommer, J.E., Fricke, C., Edward, D.A. \& Chapman, T. 2012. Interactions between genotype and sexual conflict environment influence transgenerational fitness in Drosophila melanogaster. Evolution 66: 517-531.

Carvalho, G.B., Kapahi, P., Anderson, D.J. \& Benzer, S. 2006. Allocrine Modulation of feeding behavior by the sex peptide of Drosophila. Curr. Biol. 16: 692-696.

Chapman, T. 2001. Seminal fluid-mediated fitness traits in Drosophila. Heredity 87: 511-521.

Chapman, T., Liddle, L.F., Kalb, J.M., Wolfner, M.F. \& Partridge, L. 1995. Cost of mating in Drosophila melanogaster females is mediated by male accessory gland products. Nature 373: $241-244$.

Chapman, T., Miyatake, T., Smith, H.K. \& Partridge, L. 1998. Interactions of mating, egg production and death rates in females of the Mediterranean fruit fly, Ceratitis capitata. Proc. R. Soc. Lond. B 265: 1879-1894.

Chapman, T., Neubaum, D.M., Wolfner, M.F. \& Partridge, L. 2000. The role of male accessory gland protein Acp36DE in sperm competition in Drosophila melanogaster. Proc. $R$. Soc. Lond. B 267: 1097-1105.

Chapman, T., Arnqvist, G., Bangham, J. \& Rowe, L. 2003. Sexual conflict. TREE 18: 41-47.

Chippindale, A.K., Gibson, J.R. \& Rice, W.R. 2001. Negative genetic correlation for adult fitness between sexes reveals ontogenetic conflict in Drosophila. Proc. Natl. Acad. Sci. USA 98: $1671-1675$.

Cognigni, P., Bailey, A.P. \& Miguel-Aliaga, I. 2011. Enteric neurons and systemic signals couple nutritional and reproductive status with intestinal homeostasis. Cell Metab. 13: 92-104.

Conte, R., Nodari, R.O., Vencovsky, R. \& dos Reis, M.S. 2003. Genetic diversity and recruitment of the tropical palm, Euterpe edulis Mart., in a natural population from the Brazilian Atlantic Forest. Heredity 91: 401-406.

Crawley, M.J. 2012. The R Book, 2nd edn. John Wiley \& Sons, West Sussex, UK.

Droney, D.C. 2003. Females lay fewer eggs for males with greater courtship success in a lekking Drosophila. Anim. Behav. 65: 371-378.

Edward, D.A., Fricke, C., Gerrard, D.T. \& Chapman, T. 2011. Quantifying the life-history response to increased male exposure in female Drosophila melanogaster. Evolution 65: 564-573.

Filice, D.C.S. \& Long, T.A.F. 2016. Genetic variation in maleinduced harm in Drosophila melanogaster. Biol. Lett. 12: 20160105.

Franke, E.S., Babcock, R.C. \& Styan, C.A. 2002. Sexual conflict and polyspermy under sperm-limited conditions: in situ evidence from field simulations with the freespawning marine echinoid Evechinus chloroticus. Am. Nat. 160: $485-496$.

Friberg, U. \& Arnqvist, G. 2003. Fitness effects of female mate choice: preferred males are detrimental for Drosophila melanogaster females. J. Evol. Biol. 16: 797-811.

Fricke, C., Green, D., Mills, W.E. \& Chapman, T. 2013. Agedependent female responses to a male ejaculate signal alter demographic opportunities for selection. Proc. R. Soc. Lond. B 280: 20130428.

Gavrilets, S. 2014. Is sexual conflict an "Engine of speciation"?. Cold Spring Harb. Perspect. Biol. 6: a017723.

Gavrilets, S. \& Hayashi, T.I. 2005. Speciation and sexual conflict. Evolution 59: 696-699.

Gavrilets, S. \& Waxman, D. 2002. Sympatric speciation by sexual conflict. Proc. Natl. Acad. Sci. USA 99: 10533-10538.

Härdling, R. \& Bergsten, J. 2006. Nonrandom mating preserves intrasexual polymorphism and stops population differentiation in sexual conflict. Am. Nat. 167: 401-409.

Hayashi, T.I., Vose, M. \& Gavrilets, S. 2007. Genetic differentiation by sexual conflict. Evolution 61: 516-529.

Heifetz, Y., Lung, O., Frongillo, E.A. Jr \& Wolfner, M.F. 2000. The Drosophila seminal fluid protein Acp26Aa stimulates release of oocytes by the ovary. Curr. Biol. 10: 99-102. 
Herndon, L.A. \& Wolfner, M.F. 1995. A Drosophila seminal fluid protein, Acp26Aa, stimulates egg laying in females for 1 day after mating. Proc. Natl. Acad. Sci. USA 92: 1011410118.

Holland, B. \& Rice, W.R. 1998. Chase-away sexual selection: antagonistic seduction versus resistance. Evolution 52: 1-7.

Kokko, H. \& Brooks, R. 2005. Sexy to die for? Sexual selection and the risk of extinction. Ann. Zool. Fenn. 40: 207-219.

Kołodziejczyk, M. \& Radwan, J. 2003. The effect of mating frequency on female lifetime fecundity in the bulb mite, Rhizoglyphus robini (Acari: Acaridae). Behav. Ecol. Sociobiol. 53: 110-115.

Konior, M., Radwan, J. \& Kołodziejczyk, M. 2001. Polyandry increases offspring fecundity in the bulb mite. Evolution 55 : 1893-1896.

Konior, M., Radwan, J., Kołodziejczyk, M. \& Keller, L. 2006. Strong association between a single gene and fertilization efficiency of males and fecundity of their mates in the bulb mite. Proc. R. Soc. Lond. B 273: 309-317.

Liu, H. \& Kubli, E. 2003. Sex-peptide is the molecular basis of the sperm effect in Drosophila melanogaster. Proc. Natl. Acad. Sci. USA 100: 9929-9933.

Łukasik, P., Kozielska, M. \& Radwan, J. 2010. The effect of a phosphogluconate dehydrogenase genotype on sperm competitiveness in the bulb mite, Rhizoglyphus robini. In: Trends in Acarology (M. Sabelis \& J. Bruin, eds), Springer, Dordrecht.

Lung, O., Tram, U., Finnerty, C.M., EipperiMains, M.A., Kaln, J. \& Wolfner, M.F. 2002. The Drosophila melanogaster seminal fluid protein Acp62F is a protease inhibitor that is toxic upon ectopic expression. Genetics 160: 211-224.

Murray, R., Mayes, P.A., Rodwell, V.W. \& Granner, D.K. 2003. Harper's Biochemistry, 26th edn. McGraw-Hill Companies, New York, NY.

Neubaum, D.M. \& Wolfner, M.F. 1999. Mated female Drosophila melanogaster require a seminal fluid protein Acp36DE, to store sperm efficiently. Genetics 153: 845-857.

Parker, G.A. 1979. Sexual selection and sexual conflict. In: Sexual Selection and Reproductive Competition in Insects (M.S.N.A.B. Blum, ed), pp. 123-166. Academic Press, New York.

Parker, G.A. \& Partridge, L. 1998. Sexual conflict and speciation. Philos. Trans. Royal Soc. Lond. B. 353: 261-274.

Priest, N.K., Galloway, L.F. \& Roach, D.A. 2008. Mating frequency and inclusive fitness in Drosophila melanogaster. Am. Nat. 171: 10-21.
Promislow, D.E.L. 2003. Mate choice, sexual conflict and evolution of senescence. Behav. Genet. 33: 191-201.

R Core Team. 2017. R: A Language and Environment for Statistical Computing. R Foundation for Statistical Computing, Vienna, Austria. https://www.R-project.org/.

Radwan, J. 1996. Intraspecific variation in sperm competition success in the bulb mite: a role for sperm size. Proc. $R$. Soc. Lond. B 263: 855-859.

Radwan, J. \& Siva-Jothy, M.T. 1996. The function of postinsemination mate association in the bulb mite, Rhizoglyphus robini. Anim. Behav. 52: 651-657.

Ribeiro, C. \& Dickson, B.J. 2010. Sex peptide receptor and neuronal TOR/S6K signaling modulate nutrient balancing in Drosophila. Curr. Biol. 20: 1000-1005.

Rice, W.R. 1996. Sexually antagonistic male adaptation triggered by experimental arrest of female evolution. Nature 381: $232-234$.

Rice, W.R. 1998. Intergenomic conflict, interlocus antagonistic coevolution, and the evolution of reproductive isolation. In: Species and Speciation (D.J. Howard \& S.H. Berlocher, eds), pp. 261-270. Oxford University Press, New York.

Rice, W.R. \& Holland, B. 1997. The enemies within: intergenomic conflict, interlocus contest evolution (ICE), and intraspecific Red Queen. Behav. Ecol. Sociobiol. 41: 1-10.

Stockwell, C.A. \& Mulvey, M. 1998. Phosphogluconate dehydrogenase polymorphism and salinity in the white sands pupfish. Evolution 52: 1856-1860.

Stuglik, M.T., Babik, W., Prokop, Z. \& Radwan, J. 2014. Alternative reproductive tactics and sex-biased gene expression: the study of the bulb mite transcriptome. Ecol. Evol. 4: 623632.

Swanson, W.J., Clark, A.G., Waldrip-Dail, H.M., Wolfner, M.F. \& Aquadro, C.F. 2001. Evolutionary EST analysis identifies rapidly evolving male reproductive proteins in Drosophila. Proc. Natl. Acad. Sci. USA 98: 7375-7379.

Tilszer, M., Antoszczyk, K., Sałek, N., Zajac, E. \& Radwan, J. 2006. Evolution under relaxed sexual conflict in the bulb mite Rhizoglyphus robini. Evolution 60: 1868-1873.

Wigby, S. \& Chapman, T. 2004. Female resistance to male harm evolves in response to manipulation of sexual conflict. Evolution 58: 1028-1037.

Received 18 September 2017; accepted 9 February 2018 